\title{
Programas de Educación Parental
}

\section{Parenting Programs}

\author{
Juan Carlos Martín-Quintana \\ Universidad de Las Palmas de Gran Canaria \\ Sonia Byme \\ Universidad de La Laguna
}

\author{
M. ${ }^{a}$ Luisa Máiquez Chaves \\ Universidad de La Laguna \\ Beatriz Rodríguez Ruiz \\ Universidad de La Laguna
}

\author{
M. ${ }^{a}$ José Rodrigo López \\ Universidad de La Laguna \\ Guacimara Rodríguez Suárez \\ Universidad de La Laguna
}

\begin{abstract}
Resumen. El objetivo de este artículo es proporcionar argumentos sobre la importancia de los programas grupales de educación para fomentar las competencias parentales. Para ello, se justifica la necesidad de dicho apoyo al definir la complejidad de la tarea de ser padres en la actualidad. También nos hacemos eco de la recomendación europea sobre la utilización de programas educativos para padres como una de las medidas de apoyo para el ejercicio de la parentalidad positiva. A continuación se describen varias generaciones de programas internacionales de educación para padres, así como algunos ejemplos de programas desarrollados en nuestro entorno nacional. Se reflexiona sobre los modelos de formación en los que se basan dichos programas, se ilustran los resultados obtenidos con el programa experiencial para padres en situación de riesgo psicosocial en dos comunidades españolas y se describe un nuevo programa "Crecer felices en familia", todavía en fase de implementación. Se concluye enfatizando la importancia de la evaluación de programas para extraer información sobre su eficacia y mejorar su implementación en situaciones naturales.

Palabras clave: educación parental, parentalidad positiva, programas de educación de padres.
\end{abstract}

Summary. This paper was aimed at emphasizing the importance of using parenting programs to promote parental competences. There is a need for this support taking into account the complexity of the parenting task in our modern societies. Following the European recommendation on positive parenting, those parenting programs are considered important measures to support parents in their educational role. Forward, several generations of parenting programs at the international context were briefly described and some examples of programs within the national context, as well. This paper provides some reflection on three models of parental education, and shows the results of an experiential parenting programs addressed to parents in psychosocial risk situation in two Spanish communities. A new program "Crecer felices en familia", still in the implementation phase, was also described. As a conclusion, the paper emphasized the importance of evaluating programs in order to know more about their efficacy and to improve the way of implementation in real settings.

Keywords: parent education, positive parenting, parent education programs.

\section{Aprender a ser padres en la sociedad actual}

Ser padres es una tarea vital que marca una de las transiciones evolutivas más importantes del desarrollo adulto y cuya adecuada resolución contribuye positivamente a la madurez personal de los que la

La correspondencia sobre este artículo puede dirigirse al primer autor Juan Carlos Martín Quintana. Facultad de Formación del Profesorado. Departamento de Educación de la Universidad de Las Palmas de Gran Canaria. C/ Santa Juana de Arco, 1. C.P. 35004 Las Palmas,jmartin@dedu.ulpgc.es realizan (Hidalgo, 1998). Dicha tarea presenta una serie de características que la hacen especialmente difícil en la sociedad actual (Máiquez, Capote, Rodrigo y Vermaes, 2000; Rodrigo, Máiquez, Martín y Byrne, 2008). En primer lugar, es una tarea sometida a profundos cambios históricos y sociales. Así,

\footnotetext{
Agradecimientos: Financiado por el Ministerio de Ciencia e Innovación (TRA2009_0145) concedido a la última autora. Nuestro agradecimiento a las familias de los Servicios Sociales y a los técnicos de los mismos por su participación en los programas educativos para
} padres mencionados en el artículo. 
por ejemplo, se ha producido una enorme diversificación en el modo de llevar a cabo dicha tarea debido a la gran variedad de formas familiares que conviven en nuestra sociedad. Además, están en plena redefinición en los roles de género dentro de la familia (tanto de la pareja como de los hijos e hijas) basados en principios más igualitarios, así como, las relaciones entre padres e hijos, que han pasado de ser verticales y jerárquicas a tender a ser más horizontales y democráticas. Asimismo, los padres ya no son los únicos agentes que educan sino que el escenario educativo se ha llenado de otras voces, procedentes de otros contextos como son los medios audiovisuales o el mundo del ocio, que reclaman su cuota de influencia sobre los hijos. Es por eso que muchos padres sienten en la actualidad que tienen poco poder de influencia educativa sobre sus hijos.

En segundo lugar, es una tarea que se construye y despliega en escenarios socioculturales. Ello significa que los padres aprenden esta tarea no como un ejercicio de aprendizaje formal, sino inmersos en un entramado de relaciones interpersonales mientras que realizan una serie de actividades significativas para la cultura o grupo social al que pertenecen. Ahora bien, más allá de la mera reproducción de los patrones culturales de lo que significa ser padre o madre en el grupo social de referencia, los padres hacen suya esta tarea al individualizarla y dotarla de significados personales. Ello requiere un gran esfuerzo personal e incrementa la variedad de interpretaciones actuales sobre la tarea de ser padres.

En tercer lugar, se trata de una tarea compleja porque se despliega en diferentes niveles de actuación, desde el nivel estratégico de organización del escenario educativo hasta el nivel táctico de actuaciones concretas. Requiere además flexibilidad para adaptarse a numerosas situaciones cotidianas, así como tener conciencia de cómo se está llevando a cabo para poder modificarla; ello implica que la tarea requiere un esfuerzo cognitivo y no puede llevarse a cabo basándose en "recetas" aprendidas. Volveremos sobre éste último aspecto porque tiene importantes repercusiones sobre la intervención familiar.

Por último, la tarea requiere de aliados y de apoyos sociales para llevarla a cabo. Desde luego las necesidades de apoyo, tanto informal como formal, son diferentes dependiendo tanto de las característi- cas de los padres, de las necesidades educativas de los hijos e hijas y del entorno ecológico de la familia caracterizado por tener más o menos factores de protección y de riesgo. Además, las necesidades de apoyo se van a agudizar en momentos de transición o de crisis cuando la familia tiene que hacer frente a situaciones estresantes.

Por todo ello, el Consejo de Europa, consciente de la complejidad y de las necesidades de apoyo que conlleva dicha tarea, ha promovido la Recomendación Rec (2006), sobre políticas de apoyo al ejercicio positivo de la parentalidad. Según ésta, el objetivo de la tarea de ser padres es el de promover relaciones positivas entre padres e hijos, fundadas en el ejercicio de la responsabilidad parental, para garantizar los derechos del niño en el seno de la familia y optimizar el desarrollo potencial del niño y su bienestar. La Recomendación se propone conseguir que los Estados miembros sean conscientes de la necesidad de proporcionar a los padres los mecanismos de apoyo suficientes para cumplir sus importantes responsabilidades en la crianza y educación de sus hijos. Concretamente, los Estados miembros están llamados a apoyar a los padres en sus tareas educativas a través de: (a) las políticas familiares adecuadas que proporcionen las medidas legislativas, administrativas y financieras para crear las mejores condiciones posibles para la educación positiva,(b) la prestación de servicios de apoyo para padres tales como servicios de asesoramiento locales, líneas telefónicas de ayuda y programas educativos para padres, y (c) proporcionar servicios especializados para los padres en situación de riesgo para prevenir el desplazamiento innecesario de los niños del hogar familiar motivado por situaciones de maltrato. Crear las condiciones adecuadas significa también tomar medidas para eliminar los obstáculos a la parentalidad positiva, como son las políticas para promover una mejor conciliación de la vida familiar y laboral. En este artículo nos vamos a hacer eco de estas propuestas, describiendo los programas de educación para padres, los modelos de formación en qué se basan e ilustrando sus resultados mediante algunos ejemplos.

\section{Tipos de programas de educación parental}

¿Qué entendemos por formación de padres y de madres? Según Boutin y Durning (1997) la forma- 
ción parental constituye una acción educativa de sensibilización, de aprendizaje, de entrenamiento y/o de clarificación de los valores, las actitudes y las prácticas de los padres en la educación de sus hijos. Ello propicia un proceso de desarrollo individual tendente a perfeccionar las capacidades de sentir, de imaginar, de comprender, de aprender y de utilizar unos conocimientos para la realización de la tarea de ser padre. También Vila (1998) considera que la formación de padres es un conjunto de actividades voluntarias de aprendizaje por parte de los padres y las madres que tienen como objetivo proveerles de modelos adecuados de prácticas educativas en el contexto familiar y/o modificar y mejorar las prácticas existentes, todo ello con el objeto de promover comportamientos positivos en los hijos y las hijas y erradicar los que se consideran negativos. Por su parte, Máiquez, Rodrigo, Capote y Vermaes (2000) plantean que la formación de padres debe considerar a éstos como adultos en proceso de desarrollo, que necesitan apoyo y asistencia para poder realizar su tarea educativa. Para estos autores, la formación de padres debe promover tanto las habilidades educativas de los padres y las madres como las centradas en promover la competencia personal e interpersonal, porque ambas contribuyen al desarrollo y la educación de los hijos y las hijas (Martín, 2005).

Los programas de educación parental se pueden clasificar en dos grupos: a) los que son de acceso universal, dirigidos a todos los padres y las madres y diseñados para informar y prepararlos en sus deberes y responsabilidades, y b) los que son de acceso limitado, que en su mayor parte están dirigidos a un determinado tipo de familias, en su mayoría en situación de riesgo para el menor o para la estabilidad familiar (Martín, 2005). Los primeros, los de acceso universal, pueden ser considerados de prevención primaria, ya que su objetivo es ofrecer un servicio a un buen grupo de familias con la intención de prevenir retrasos en el desarrollo y pautas educativas inadecuadas. Un ejemplo de este tipo de programas puede ser el que ofrece un servicio de cuidados prenatales y clases de educación a todas las madres primerizas. Otro ejemplo es el de programas de formación de padres y madres, las llamadas "Escuelas de padres" que pretenden informar y formar a un gran número de padres y de madres a través de reuniones en Centros Educativos. Por su parte, los programas específicos de acceso limitado, están dirigidos a personas y familias que cumplen con una serie de criterios para ser incluidos dentro de grupos en riesgo bio-psico-social. Las personas de estos grupos tienen mayor probabilidad de manifestar problemas en su desarrollo o en el de sus hijos e hijas, si no se realiza ninguna intervención. Estos programas específicos pueden estar dirigidos a padres y madres con nivel educativo y económico bajo; para la prevención del abuso infantil y la negligencia; para la prevención de la violencia y la delincuencia juvenil; para la educación de hijos e hijas con discapacidades o con problemas de conducta y para la educación parental de las madres adolescentes, entre otros.

A continuación resumimos algunos de los programas de educación parental, tanto universales como selectivos y basados tanto en visitas domiciliarias como en la atención grupal. Estos programas han ido evolucionando a lo largo del tiempo cambiando sus objetivos, metodología y contenidos según el enfoque conceptual y las necesidades de los padres y las madres de cada generación. En los siguientes cuadros 1,2 y 3 . describiremos tres generaciones de programas de educación parental según Rodrigo et al. (2008).

\section{Programas de primera generación}

Estos programas sitúan el énfasis en la calidad de las pautas educativas parentales. Concretamente pretenden, entre otros logros, que los padres y las madres proporcionen un ambiente de apoyo y estimulación para el desarrollo de los niños y las niñas; que conozcan el desarrollo evolutivo e incrementen su propia competencia; que desarrollen estrategias de comunicación efectivas y de resolución de problemas; que fomenten el desarrollo cognitivo, linguiístico, social y emocional en los niños y las niñas. Para ello, promueven el contacto con otros padres en sesiones de grupo o se basan en visitas domiciliarias (Rodrigo, 2003). Corresponden cronológicamente a los años setenta y responden a enfoques unidireccionales, en los que se interviene sólo con los padres y las madres para que sus efectos repercutan positivamente en la educación de sus hijos e hijas. 
Cuadro 1. Primera generación: calidad de las pautas educativas parentales

\section{Objetivos}

- Proporcionar un ambiente de apoyo y de estimulación para el desarrrollo del niño.

- Conocer el desarrollo evolutivo e incrementar su propia competencia.

- Entrenar en estrategias de comunicación efectivas y de resolución de problemas.

- Entrenar en estrategias de desarrollo cognitivo, lingüístico, social y emocional en los niños.

- Promover pautas educativas saludables y prevenir el maltrato.

- Promover el contacto con otros padres en sesiones de grupo.

- Basarse también en visitas domiciliarias.

\section{Programas de segunda generación}

Los programas de segunda generación sitúan el énfasis en la calidad de la interacción padres-hijos durante la realización de actividades cotidianas para construir pautas positivas de apego; promover la sensibilidad parental, la empatía hacia las necesidades de los hijos y las hijas y el afecto; enseñan cómo poner límites, cómo manejar las conductas inadecuadas y jugar con los hijos de manera efectiva y positiva y aprender a usar estrategias de interacción con menores agresivos. Todo esto se realiza mediante el uso de viñetas, videos, episodios y se fomentan las reuniones con otros padres y madres para el apoyo social. Estos programas de segunda genera-

\section{Programas}

- Sistematic Training for Effective Parenting (Dinkmeyer y McKay, 1976): STEP.

- Parent Effectiveness Training (Gordon, 1980): PET.

- Minnesota Early Learning Design (Ellwood, 1988): MELD.

- Early Childhood Family Education (Minnesota Departamento of Education, 1975): ECFE.

- Home Instruction Program for Preschool Youngsters (Lombard, 1981): HIPPY

- Parents as Teachers (Winter y Rouse, 1981): PAT.

- Nurse Family Partnership (Olds, Henderson, Chamberlin y Tatelbaum, 1986).

\section{Programas de tercera generación}

Los programas de tercera generación que se están experimentando en la actualidad, tienen como objetivo fundamental fomentar la calidad del funcionamiento familiar como sistema, mediante intervenciones comprehensivas duraderas, multi-dominio y multi-contexto (Rodrigo, 2003). Los objetivos son fomentar la relación de pareja y la co-parentalidad; apoyar la transición a la maternidad de las madres adolescentes o jóvenes con bajos ingresos económi-

Cuadro 2. Segunda generación: calidad de la interacción padres-hijos

\section{Objetivos}

- Mejorar la interacción durante actividades cotidianas rutinarias para construir pautas positivas de apego.

- Promover la sensibilidad parental, la empatía hacia las necesidades de los hijos mostrar el efecto.

- Enseñar cómo poner límites, manejar las conductas inadecuadas y jugar con los hijos de manera efectiva y positiva.

- Aprender a usar estrategias de interacción con niños agresivos y con problemas de conducta.

- Uso de viñetas, vídeos, episodios.

\section{Programas}

- Steps Howard effective Enjoyable Parenting (Egeland y Erikson, 1993, Minnesota): STEEP.

- Famuly development service program (Heinicke, 1991, UCLA).

- The incredible years (Webster-Stratton 1992).

- Oregon Social Learning Center (Patterson, 1974), OSLC.

- Positive parenting program, Triple P (Sanders, 1999, Australia: Queesland). 
Cuadro 3. Tercera generación: calidad del funcionamiento familiar como sistema

\section{Objetivos}

- Intervenciones comprehensivas, duraderas, multidominio y multicontexto.

- Relación pareja y coparentabilidad.

- Transición maternidad: madres adolescentes o jóvenes de bajos ingresos.

- Promover las competencias parentales y el apoyo familiar.

- Dos generaciones: educación para padres con bajos ingresos: educación temprana hijos, alfabetización padres y habilidades parentales.

- Intervenciones en la familia y en la escuela para prevenir la conflictividad y los problemas de conducta.

cos. También se pueden encontrar en este grupo experiencias de participación familia-escuela con el fin de prevenir las conductas agresivas y mejorar la convivencia.

En el siguiente cuadro 4, sin pretender ser exhaustivos, hemos incluido algunos programas que se llevan a cabo en España en la actualidad, que están sistematizados como tales, y están dotados de un sistema de evaluación.

\section{Programas}

- Becoming a family Project (Cowan y Cowan, 1992).

- Family nurturing center (Bavolek, 2002).

- ADVANCE (Webster-Stratton, 1994)

- NEW CHANCE intervention (Quint y Engeland, 1995).

- Family and School Together (Coie, Watt, West, Hawkins, Markman, Ramey, Shure y Long, 1993); FAST TRACK.

- Early Alliance program (Dumas, Prinz, Smith y Laughlin, 1999)

\section{Modelos de formación de padres}

Los primeros programas de educación de padres surgieron en la tradición de las llamadas Escuelas de Padres que se llevaban a cabo, por lo general, en centros educativos. Todavía es frecuente utilizar como sinónimo de formación de padres esta denominación de Escuelas de padres. Sin embargo, como veremos, estas escuelas se basaban en un modelo

Cuadro 4. Algunos ejemplos de programas de padres en España

\section{Programas}

- Programa de Apoyo a Madres y Padres de Adolescentes. Sevilla: Oliva, Hidalgo, Parra, Ríos y Vallejo, (2007)

- La Corresponsabilidad Familiar (COFAMI): fomentar la cooperación y la responsabilidad de los hijos. País Vasco: Maganto y Bartau (2004).

- Preescolar Na Casa. Galicia, Equipo de Preescolar Na Casa, (1996).

- Programa-Guía para el desarrollo de competencias emocionales, educativas y parentales, Martínez-González, (2009).

- Programa Comunicación cooperativa entre la familia y la escuela. Valencia, Forest y García-Bacete, (2006).

- Programa de Apoyo Personal y Familiar en colaboración con Radio ECCA Fundación Canarias. Rodrigo, Capote, Márquez, Martín, Rodríguez, Guimerá y Peña (2000)

- Crecer Felices en Familia: Un Programa de Apoyo Psicoeducativo para Promover el Desarrollo Infantil, Junta de Castilla y León. Rodrigo, Máiquez, Byrne, Rodríguez, Martín, Rodríguez y Pérez (2008).

\section{Centros donde se dispensan}

- Centros escolares.

- Servicios Sociales.

- Centros escolares y servicios sociales.

- Familias y Centros escolares.

- Centros escolares y Servicios sociales.

- Centros escolares.

- Servicios sociales y centros escolares de atención preferente.

- Servicios sociales. 
bastante cuestionado en la actualidad, tendente a identificar la formación de padres con un proceso académico de educación formal, basado en el aprendizaje de conocimientos. Este no es el único modelo de formación como tendremos ocasión de comprobar. A continuación analizaremos los diferentes modelos de formación de padres y madres, como son el modelo académico, el técnico y el experiencial (Máiquez et al., 2000).

\section{El modelo académico}

Este modelo consiste en la adquisición de conceptos sobre el desarrollo y la educación de los hijos e hijas en un escenario de aprendizaje formal. Como tal, se basa en la transmisión de contenidos teóricos sobre psicología evolutiva y educativa, ejemplificándolos adecuadamente para que los padres los entiendan. Esta información estaría centrada en el "deber ser", esto es, en lo que los padres deben hacer para favorecer el desarrollo y la educación de sus hijos e hijas. Se parte del supuesto de que los padres bien informados tenderán fácilmente hacia un cambio de actitud con respecto al desarrollo y la educación de sus hijos. Sin embargo, la información es necesaria pero no suficiente para el cambio. Para que una actitud cambie, el conocimiento se tiene que conectar con las experiencias previas, darle un valor de verdad y percibir que se obtendrá un beneficio por ese cambio. Si esto no se cumple, no se puede esperar un cambio de actitud. Nuestra vida cotidiana está llena de ejemplos: ¿cuántas veces no se han planteado muchos fumadores la posibilidad de dejar de fumar, de cambiar su actitud ante el consumo de tabaco y no lo hacen? No será porque no tienen información suficiente sobre las consecuencias negativas que tiene el consumo de esta sustancia para la salud. Pues bien, hasta que esa persona no conecte con alguna experiencia personal o cercana en la que perciba claramente el daño que le ocasiona el consumo de tabaco, y hasta que no tome conciencia de los beneficios personales y sociales que puede obtener por dejar de fumar, no creerá que el consumo de tabaco es perjudicial para su salud y no cambiará por tanto su actitud. En el contexto familiar ocurre lo mismo. Los padres solemos estar informados de lo que deberíamos hacer para mejorar el desarrollo y la educación de nuestros hijos e hijas, pero cuando llegamos a casa, nuestra realidad sociofamiliar y nuestras creencias implícitas se anteponen y seguimos actuando de la misma manera.

Este modelo puede generar además frustración y sentimiento de culpabilidad en los padres. El "deber ser" nos recuerda constantemente lo buen o mal padre o madre que somos. En la mayoría de las veces, al contrastar la información con lo que hacemos cotidianamente, vamos tomando conciencia de todo aquello que podríamos hacer, pero que por muchas causas, no lo realizamos. Es lógico que ante esta situación los padres se puedan sentir frustrados, con sentimientos de culpabilidad o de baja capacidad en su tarea educativa. Por otro lado, cuando la formación de los padres se basa en el modelo académico, la evaluación suele dirigirse bien a recoger el nivel de conocimientos adquiridos por éstos, o bien a la capacidad del experto en la transmisión de la información. Ninguno de estos dos objetivos apunta en la dirección de una evaluación del cambio de actitud y comportamiento de los padres y las madres. En suma, este modelo tradicional de formación académica olvida, entre otras cosas, que los padres acuden a los programas con unas teorías implícitas relativas a multitud de temas que, aunque no sean conscientes de ellas, actúan como filtros a través de los cuales tamizan toda la información que reciben y dan lugar a variadas interpretaciones sobre un mismo contenido (Máiquez et al., 2000).

\section{El modelo técnico}

Los programas que se sitúan en este modelo pretenden que los padres y las madres adquieran técnicas y procedimientos basados en la modificación de conducta, en el aprendizaje social, u en otros modelos, en un escenario de aprendizaje experto. Esta segunda modalidad en la formación de los padres y las madres se inspira en la imagen de éstos como técnicos eficaces (Máiquez et al., 2000). A diferencia de la modalidad académica, se pretende que a los padres no se les proporcione tanta información general, sino que se les enseñen técnicas específicas de control de la conducta de los hijos. El experto 
entrena a los padres y a las madres en toda una batería de técnicas como refuerzos, castigos, retirada de privilegios, modelado, etc., ayudándoles a modelar su conducta ante estos casos.

Según los autores que defienden este modelo, tiene la ventaja de poder entrenar a varias personas en un breve espacio de tiempo, con lo que se potencia el impacto terapéutico con un mínimo de profesionales (Polaino-Lorente y Cerezo, 1984). No obstante, este tipo de modelo de formación puede fomentar la dependencia del técnico. Los padres pueden aprender a manejar una serie de técnicas, como si fueran verdaderas "recetas", pero no conocen su utilidad o su sentido y significado en el contexto sociocultural (Brown, Collins y Duguid, 1995). Esto puede ocasionar que el uso de estas técnicas no consiga los objetivos deseados lo que les llevará a acudir una y otra vez ante el técnico para solicitar qué hacer. Asimismo, con este modelo se puede ir perdiendo la credibilidad de los expertos. Llegará un momento en el que los padres y las madres, que no perciban resultados positivos tras el uso de las técnicas propuestas, o bien renuncien a seguir intentándolo o bien se rebelen contra el técnico. Indicadores de que esto está ocurriendo lo podemos encontrar en las siguientes verbalizaciones de los padres y las madres: "una cosa es lo que dice la teoría y otra es la práctica"; "en el papel todo resulta muy bonito", "con usted todo parece muy sencillo, pero cuando llego a mi casa nada es igual", etc.

Como señalan Graziano y Diament (1992), los padres necesitan participación "práctica", que se centre sobre situaciones y procedimientos específicos que son más efectivos que los acercamientos de "clase o aula" y de conocimientos generales. Pero como ocurre en el modelo académico, no se tiene en cuenta las creencias o ideas previas de los padres y las madres, y como decíamos anteriormente, los padres y las madres participan activamente pero sólo en lo que es la reproducción de una serie de técnicas descontextualizada de lo que es su situación sociofamiliar.

\section{El modelo experiencial}

Los programas que se sitúan en este modelo pretenden llevar a cabo una reconstrucción del conoci- miento episódico cotidiano en un escenario sociocultural. No se trata de una divulgación de contenidos teóricos, ni tampoco de un entrenamiento de determinadas técnicas, se trata de una formación de padres y de madres de tipo experiencial, que tenga como objetivo primordial conceptualizar las prácticas de la vida cotidiana. Esto supone que la tarea principal que van a realizar los padres y las madres es identificar, reflexionar y analizar sus propias ideas, sentimientos y acciones en los episodios de la vida cotidiana. Van a partir de lo que ya realizan, de sus prácticas educativas, no de lo que se debería hacer. En este modelo, a diferencia de los dos anteriores, se tienen en cuenta las creencias o ideas previas de los padres y las madres, ya que a partir de éstas y de las situaciones cotidianas, y mediante un proceso inductivo de construcción del conocimiento cotidiano-experiencial, van a ir construyendo su conocimiento (Máiquez et al., 2000). Ahora bien, esta construcción del conocimiento, se ve favorecida por el escenario de la intervención. Estamos hablando de un proceso que se realiza por medio de sesiones grupales con otros padres o madres, por lo que se favorece la construcción compartida del conocimiento. La gran variedad de experiencias que se exponen en cada una de las sesiones, hace que los padres y las madres puedan contrastarlas con las suyas propias.

En este modelo, a diferencia de los dos anteriores, es difícil que se propicien en los padres y las madres sentimientos de culpabilidad, frustración, dependencia e incompetencia parental. La razón de ello es que al partir de episodios de la vida cotidiana, facilitando la reflexión y análisis de lo que ya hacen y propiciando el que formulen compromisos de cambio en función al resultado de sus análisis, estamos fomentando el que los padres tengan una participación activa en todo el proceso y sobre todo, que sean sus propios agentes activos de cambio. Este modelo invita a la acción, bien reforzando lo que vienen haciendo en pro de la educación y desarrollo de sus hijos e hijos y de la relación familiar, o bien retomando su papel como educadores, en aquellas situaciones educativas en las que perciban que deben tener una mayor participación (Martin, 2005).

Una de las variables a evaluar en los programas experienciales y que ayuda a entender las diferencias de los tres modelos expuestos es la percepción 
sobre la dificultad del rol parental. Desde el modelo académico, esta dificultad puede provenir después de mucha información conceptual sobre todo lo que deberíamos hacer como padre o madre, en la que éstos se ven ante un mundo que les apabulla, pero desarmados, sin recursos para afrontar esta difícil tarea. En el modelo técnico, esta percepción de la dificultad en la tarea de ser padre o madre, puede surgir tras recibir un amplio entrenamiento en técnicas de modificación de conducta, donde se ven con el conocimiento del uso de cada una de ellas, pero muy desorientados por no saber cuándo llevarlas a cabo. En el modelo experiencial, esta dificultad surge de la toma de conciencia de los padres y las madres de su propia realidad sociofamiliar, pero que gracias a la observación de alternativas que facilitan las experiencias de otros padres y al análisis de las consecuencias que realizan sobre su propia forma de actuar, hacen que se encuentren con los recursos suficientes para asumir compromisos de cambio y para afrontar con mayor efectividad las situaciones familiares conflictivas (Martín, 2005).

\section{Resultados de la evaluación de un programa experiencial}

Uno de los programas para padres y madres en riesgo psicosocial diseñado por algunos de los autores de este artículo y que sigue la metodología experiencial es el "Programa de Apoyo Personal y Familiar". Ha sido elaborado por el equipo de intervención familiar del Departamento de Psicología Evolutiva y de la Educación de la Universidad de La Laguna y Radio ECCA Fundación Canaria. Entre sus objetivos generales está el que los padres y las madres retomen o refuercen su papel como educadores, mediante prácticas de reflexión y análisis personal sobre el desarrollo y la educación de los hijos y las hijas, además de proporcionar situaciones en las que adquieran y descubran los recursos personales y sociales necesarios para afrontar las situaciones adversas que pueden presentarse tanto a nivel personal o social como en la educación de sus hijos. Asimismo, este programa cuida mucho la integración social de las familias, teniendo en cuenta que el apoyo social es un factor de protección muy importante. Se han elaborado seis módulos de habilidades parentales que pretenden dar respuestas a las necesidades de los padres y de los menores, y las problemáticas familiares que más inciden en el ejercicio de la parentalidad. Cada módulo contiene, entre 4 a 7 sesiones, siendo 31 el total de sesiones de las que consta, de las cuáles dos son iniciales para ayudar a conformar la estructura grupal y dar tiempo a que el grupo se vaya configurando. Cada una de estas sesiones se realiza semanalmente, con una duración de hora y media; de esta manera, el programa se puede llevar a cabo a lo largo de un curso escolar. Estos módulos son: Organización de las actividades cotidianas; Los padres y las madres ante los problemas del desarrollo; Comunicación y resolución de conflictos en la familia; El afecto en la familia; Situaciones que cambian la vida familiar; y Educar en la adolescencia.

En la evaluación de este programa en Canarias participaron 340 madres (185 habían realizado el programa y 155 constituyeron el grupo control) referidas por los Servicios Sociales municipales de la isla de Tenerife. En dicha evaluación, se observaron cambios significativos en los tres grandes grupos de variables medidas: las ideas sobre el desarrollo y la educación de los hijos e hijas, la percepción de la agencia personal y las prácticas educativas (Martín, 2005; Martín, Máiquez, Rodrigo, Correa y Rodríguez, 2004). Al finalizar el programa, las madres se manifestaron menos favorables a las teorías que obstaculizan el desarrollo y la educación de los hijos e hijas (teorías Nurturistas e Innatistas). También el programa consiguió cambios significativos en las madres en cuanto a la agencia personal, esto es, informaron de mayor Auto-eficacia, Locus de Control Interno, Dificultad en el rol parental y Acuerdo marital. Así mismo, modificaron sus prácticas educativas siendo menos Permisivas-Negligentes y menos Coercitivas, al tiempo que utilizaron más las prácticas Inductivas basadas en la argumentación y negociación con sus hijos e hijas (Martín et al. 2004; Rodrigo, Correa, Máiquez, Martín, y Rodríguez, 2006; Rodrigo, Máiquez, Correa, Martín y Rodríguez, 2006). La mayoría de estos cambios fueron significativos respecto al grupo de control.

Actualmente, se está llevando a cabo una nueva evaluación del programa en su implementación comunitaria en la mayoría de las corporaciones loca- 
les de la Junta de Castilla y León, con la participación de unos mil padres y madres, la mitad de los cuales terminaron el programa y los restantes como grupo de espera del programa en su siguiente edición que hace las veces del grupo de control. El programa de la Junta de Castilla y León se denomina "Educar en familia" y está basado en el programa llevado a cabo en Canarias. En esta nueva edición, además de cambios en sus contenidos y formatos de presentación, se han ampliado las áreas de evaluación, incluyendo los cambios en las redes de apoyo formal e informal de los participantes y los cambios en función del nivel de riesgo de los padres y de la naturaleza del grupo (número, carácter o no mixto y formación del mediador). Asimismo, en una muestra extraída al azar de aquellos que terminaron el programa se ha evaluado por primera vez el impacto del programa en el ambiente del hogar un año después, medido objetivamente por medio de una empresa de evaluadores contratada al efecto y según las opiniones de los propios técnicos. Además, se evaluó el impacto del programa en el desarrollo profesional de los mediadores, su nivel de "burnout" y el impacto del programa en el servicio de atención a las familias y los menores en riesgo. Todo ello nos ayudará a mejorar la formación de los mediadores y la forma de implementación del programa. Los primeros resultados indican que los cambios más notables se han obtenido en el cambio de teorías implícitas y en las prácticas educativas, en la misma línea que el programa llevado a cabo en Canarias, mientras que en los factores de agencia parental se han manifestado cambios positivos en el locus de control y el acuerdo en la pareja. Los cambios en el programa reportados por los padres predijeron la calidad del ambiente familiar observada al año de terminar el programa lo que indica una buena estabilidad en sus efectos a largo plazo. En sucesivas publicaciones daremos debida cuenta de todos estos resultados.

\section{Un nuevo programa "Crecer Felices en Familia" para el apoyo psicoeducativo de padres y promover el desarrollo infantil}

Actualmente se está llevando a cabo un nuevo programa de educación parental, "Crecer Felices en
Familia”, que sigue la metodología experiencial y que describimos a continuación en sus líneas fundamentales. El programa "Crecer Felices en Familia: programa de apoyo psicoeducativo para promover el desarrollo infantil" (Rodrigo, Máiquez, Byrne, Rodríguez, Martín, Rodríguez y Pérez, 2009), forma parte de una de las acciones psicoeducativas y comunitarias que varias Instituciones: Gerencia de los Servicios de Protección de Menores de la Junta de Castilla y León, Departamento de Psicología Evolutiva y de la Educación de la Universidad de La Laguna y el Departamento de Educación de la Universidad de Las Palmas de Gran Canaria, llevan realizando con las familias usuarias de los Servicios Sociales en situación de riesgo psicosocial.

\section{Características del programa}

El programa se caracteriza por estar centrado en promover las competencias de los padres y promover el desarrollo de los niños desde su nacimiento hasta los 5 años. Con su implementación se estimula la reflexión sobre las cogniciones de los padres respecto al comportamiento con los hijos. Tiene una orientación muy práctica dirigida al entrenamiento de pautas concretas de actuación y secuencias de interacción, inspirándose en la metodología experiencial. Un aspecto importante es que el mediador tiene una función de acompañamiento, asesoramiento, apoyo y modelaje para los padres. Sigue un esquema de colaboración con los padres mediante el refuerzo de sus capacidades educadoras y de su implicación en la tarea. Por lo que respecta a la temporalización de la intervención, éste programa es de cinco meses aproximadamente y una hora y media semanal.

Rodrigo et al. (2008) señalan los puntos fuertes y débiles de la intervención grupal que son extensivos a este programa. Entre los fuertes está el que promueve la integración comunitaria al fomentar el uso de los recursos comunitarios disponibles; asegura una implementación más homogénea de la intervención; rompe la barrera a recursos comunitarios, en este sentido normaliza a las familias que se encuentran en situación de riesgo psicosocial y proporciona apoyo grupal, de modo que el grupo se convierte 
en una de las fuentes importantes de apoyo. Pero la atención grupal también tiene ciertos puntos débiles como son: el grupo en la metodología experiencial se convierte en un grupo experiencial y asume todas las características de éstos, siendo la participación la más importante; otro punto débil es que requiere un esfuerzo sostenido para que asistan los padres durante varios meses, máxime cuando trabajamos con familias en situación de riesgo psicosocial; y por último otro de los puntos débiles es que el mediador tiene que tener una buena formación en dinámicas de grupo. En la medida que el mediador tenga la información y formación necesaria para trabajar con padres y madres, estos puntos débiles se pueden minimizar fácilmente.

Este programa se dirige a las siguientes poblaciones: a madres y padres con historia de negligencia o maltrato físico y/o emocional; madres adolescentes y/o con embarazos no deseados; madres y padres con escasas capacidades parentales y/o que tienen hijos con problemas de salud, y/o temperamento difícil; madres y padres con problemas de salud, con inestabilidad emocional y/o ligero retraso mental; madres y padres con un nivel socioeconómico bajo combinado con condiciones de riesgo y vulnerabilidad social y madres y padres de población inmigrante.

\section{Objetivos}

Los objetivos del programa son los siguientes:

- Promover el desarrollo de apegos seguros.

- Animar a los padres a reconocer y reaccionar positivamente ante los progresos evolutivos de los hijos.

- Enseñar habilidades básicas de cuidado y seguridad infantil.

- Ayudar a los padres a identificar y responder a las necesidades de los hijos.

- Ayudar a los padres a distinguir y entender los estados mentales de los hijos y promover su autonomía física y emocional.

- Ayudar a los padres a identificar los intentos de comunicación verbal y no verbal de los hijos.

- Animar a los padres a que hablen a sus hijos y respondan a sus verbalizaciones.

- Enfatizar la riqueza cognitiva y afectiva del juego y la resolución de tareas conjuntas padres e hijos.

- Orientar a los padres sobre la enseñanza de hábitos durante las rutinas cotidianas.

- Ofrecer pautas para la regulación del comportamiento de los hijos de acuerdo a las normas.

- Hacer conscientes a los padres sobre sus percepciones y atribuciones del comportamiento infantil y los estados emocionales que este les suscita.

- Promover la reflexión sobre las consecuencias de la propia conducta en el desarrollo de los hijos.

- Favorecer los factores de protección y reducir los de riesgo mediante la reducción del estrés, el incremento del apoyo social y la satisfacción de las necesidades de los padres a múltiples niveles.

- Fomentar el disfrute de la parentalidad y de la relación con el niño.

\section{Competencias que fomenta el programa}

El programa promueve aquellas competencias que consideramos necesarias en el ejercicio de una parentalidad positiva (se pueden consultar en el artículo de Rodrigo, Martín, Cabrera y Máiquez en el mismo monográfico). De esta manera, el programa promueve entre otras habilidades educativas, la calidez en las relaciones con los hijos y reconocimiento de sus logros alcanzados en la medida de sus posibilidades; el control y supervisión del comportamiento infantil gracias a la comunicación y fomento de la confianza en sus buenas intenciones y capacidades; la estimulación y apoyo al aprendizaje y la adaptabilidad a las características del niño.

Igualmente este programa promueve habilidades de autonomía personal y búsqueda de apoyo social, la implicación en la tarea educativa al considerarse capaces para llevar a cabo el rol parental; la responsabilidad ante el bienestar del niño; el tener una visión positiva del niño y de la familia; la búsqueda de ayuda de personas significativas con el fin de complementar el rol parental en lugar de sustituirlo o devaluarlo; el acuerdo en la pareja sobre criterios educativos y los comportamientos que deben seguir- 
se con los hijos; la identificación y utilización de recursos para cubrir las necesidades como padres o madres y como adultos y el manejo adecuado del estrés parental. Por último, se promueven competencias infantiles, el desarrollo de habilidades resilientes a través de dos vías principales: el fomento de vínculos seguros y el desarrollo de estrategias de regulación personal.

\section{Estructura y contenidos del programa}

- Módulo 1: El desarrollo del vínculo afectivo.

- Módulo 2: Conocer a nuestro hijo/a.

- Módulo 3: Aprender a regular el comportamiento infantil.

- Módulo 4: La primera relación con la escuela.

- Módulo 5: Educar, ¿una tarea en solitario?

Cada uno de los módulos consta de 4 sesiones, de modo que el programa contiene 20 sesiones de hora y media de duración y con periodicidad semanal más las de formación del grupo y evaluación.

\section{La formación de mediadores}

El programa sigue la metodología experiencial que ha sido ya validada en otros programas de educación parental (Máiquez et al., 2000; Martín, 2005; Martín et al., 2004; Rodrigo et al., 2006; Rodrigo et al, 2006) y descrita someramente en el punto 3.3. En esta metodología resulta crucial el papel del profesional que no es el del experto sino el del mediador o facilitador del proceso de construcción de conocimiento.

En el modelo de experto se considera que los profesionales son los que poseen el conocimiento, lo que les otorga autoridad y poder para asumir el control absoluto y tomar todas las decisiones. Según el modelo de mediador, el protagonismo debe ser de los padres validados con sus ideas, concepciones, prácticas y su forma de ver la realidad familiar. El proceso de cambio no es lineal y de fuera a dentro sino curvilíneo y de dentro a fuera. En ningún momento hay que desautorizar la voz de los padres sino plantearles voces alternativas y cursos alternativos de actuación que deben salir del grupo o, en todo caso, iniciados o sugeridos por el mediador. Otra característica fundamental es no dar por sentado que existe un solo modelo de educar sino que hay que ayudar a los padres a encontrar su propio modelo realizando correcciones puntuales al que ya se está utilizando.

La misión del mediador tiene varias facetas. La primera es la de facilitar y mejorar las formas de verbalización que emplean los padres para referirse a las situaciones educativas, a sus concepciones y a los comportamientos de los hijos y los suyos propios. En una segunda faceta, el mediador es una fuente de información alternativa, es un miembro más del grupo que aporta ideas, creencias y prácticas con una finalidad muy clara, la de proporcionar modelos educativos alternativos que no hayan salido espontáneamente en el discurso explícito de los padres. El mediador debe apoyar y facilitar el proceso de intercambio, negociación y transformación del conocimiento episódico en el seno del grupo. El mediador debe canalizar la atención del grupo hacia los aspectos relevantes de la tarea, debe estructurarla y graduarla de modo que esté acorde con las capacidades de los padres (sus creencias y el nivel de explicitación de las mismas, su grado de conocimiento alternativo, su manejo del lenguaje, etc.), debe dar pistas indirectas que reorienten al grupo, y debe jugar con el progresivo traspaso en el control de las situaciones o tareas. Por último, es fundamental que el mediador regule el clima emocional del grupo concretamente el nivel de frustración, el autoconcepto parental, las relaciones interpersonales y los aspectos motivacionales (Máiquez et al.,2000; Rodrigo, Máiquez, Martín y Rodríguez 2006) .

Todo lo anterior implica una apuesta fuerte por la formación de los mediadores. Al inicio del programa, resulta fundamental la formación de los mediadores y personal del servicio. En esta formación, se intenta que conozcan no sólo los contenidos del programa sino también cómo deben realizar su tarea como mediadores para que sea lo más eficaz posible. Pero además, para conseguir los resultados deseados es necesario realizar un seguimiento de la implementación del programa con reuniones de seguimiento periódicas con los profesionales que lo llevan a cabo. En estas reuniones, se analizan las dificultades en la puesta en práctica de las activida- 
des propuestas en el mismo y los problemas de índole práctica que van surgiendo durante la implementación del programa (Martín, 2005; Rodrigo et al. 2006).

\section{Evaluación}

Se ha planteado un sistema de evaluación centrado en analizar los cambios en los padres y en el ambiente educativo familiar obtenidos con el programa teniendo en cuenta el nivel de riesgo psicosocial de partida y la calidad del sistema de apoyo. Además se valora el impacto del programa en el servicio y en el técnico/mediador que lo ha llevado a cabo. Asimismo, se valora a medio plazo (seis meses) el impacto del programa en la salud de los padres y del niño/a y en el uso de recursos.

Hemos utilizado una variedad de medidas relacionadas con los objetivos del programa que son las siguientes: a) Evaluación inicial sobre el contexto de riesgo psicosocial de la familia; b) Cuestionarios sobre competencias parentales a cumplimentar por los padres y cuestionarios para la evaluación del ambiente familiar a cumplimentar por el técnico que conoce a la familia (inicial y final); c) Impacto del programa en la salud de los padres y del niño según los medidores del programa y valoración del impacto del programa en el servicio y en el técnico/mediador según técnico/mediador. Este programa se está implementando desde septiembre de 2009 por lo que todavía no contamos con resultados sobre su eficacia.

\section{Conclusión}

En el recorrido realizado sobre los programas grupales para padres hemos hecho una especial mención al tema de su evaluación. Al terminar, hacemos hincapié en la misma ya que estamos convenidos de su importancia, es una asignatura pendiente para los técnicos y un reto para los investigadores y además está en consonancia con la Recomendación europea descrita al comienzo del artículo. Sin esta evaluación no puede extraerse información sobre cómo deben ser los apoyos edu- cativos a los padres para que resulten más eficaces. Los programas descritos e ilustrados en este trabajo están también dentro de las directrices marcadas en dicha recomendación para la realización de programas grupales para padres. Los programas de la línea experiencial se basan en promover el desarrollo de competencias parentales (empoderamiento) y no en el descubrimiento de sus déficits. Además, es crucial la integración del programa dentro de la red de recursos estables de la comunidad y proporcionar una buena coordinación entre los diferentes servicios de apoyo. Los programas deben estar integrados en los planes de caso y ser una fuente constante de apoyo para aquellas familias que lo necesiten. Los programas deben satisfacer las necesidades de los padres y no resultar prescriptivos como ya se ha comentado. Por último, deben proporcionar pruebas empíricas sobre su eficacia y reflejar las buenas prácticas de los profesionales. En este sentido, terminamos recordando las palabras de la recomendación europea antes aludida según las cuales los Estados miembros deben promover investigaciones más sólidas sobre la eficacia de los programas educativos para padres y validar el trabajo de apoyo que llevan a cabo los técnicos con las familias. Para ello debemos definir y consensuar los estándares apropiados para la evaluación de programas y mejorar la calidad de la formación inicial y continua de los técnicos así como definir las variables clave que garantizan su buena implementación. Todos estos aspectos hay que sistematizarlos ya que son tan cruciales para el programa como la calidad de sus contenidos.

\section{Referencias}

Boutin, G. y Durning, P. (1997). Intervenciones socioeducativas en el medio familiar. Madrid: Narcea.

Brown, J.S., Collins, A. y Duguid, P. (1995). La cognición situada y la cultura del aprendizaje. Kikiriki, 39, 46-60.

Comité de Ministros a los Estados Miembros: Recomendacion Rec 19 (2006), (http://www.coe. int/t/dg3/youthfamily ).

Graciano, A.M. y Diament, D.M. (1992). Parent behavioral training : An examination of the parad- 
ingm, Behavior Modification, 16, 3-38

Hidalgo, M.V. (1998). Transición a la maternidad y a la paternidad, en M.J. Rodrigo y J. Palacios (coords.), Familia y Desarrollo Humano. Madrid. Alianza Editorial.

Máiquez, M.L., Rodrigo, M.J., Capote, C. y Vermaes, I. (2000). Aprender en la vida cotidiana. Un programa experiencial para padres. Madrid: Visor.

Martín, J.C., (2005). Evaluación del programa de apoyo personal y familiar para familias en situación de riesgo psicosocial. Universidad de La Laguna: Tesis doctoral no publicada.

Martín, J.C.; Máiquez, Rodrigo, Correa y Rodríguez, (2004). Evaluación del programa "Apoyo personal y familiar" para madres y padres en situación de riesgo psicosocial. Infancia y aprendizaje, 27 (4), pp. 437-445.

Oliva, A., Hidalgo, V., Martín, D., Parra, A., Ríos, M. y Vallejo, R. (en prensa). Programa de Apoyo a Madres y Padres de Adolescentes. Sevilla

Polaino-Lorente, A. y Cerezo Jimenez, M.A. (1984). Algunos factores posibilitadores del desarrollo del entrenamiento de padres en modificación de conducta como una alternativa terapéutica. Revista de Psicología General y Aplicada, 39, 1140-1162.

Rodrigo, M.J. (2003). Programas de apoyo a las necesidades de los padres. Ponencia en el simposio Las relaciones padres-hijos: necesidades de apoyo a las familias. Save the Children. Madrid: Exlibris.

Rodrigo M.J. y Palacios, J. (1998). Familia y desarrollo humano. Madrid. Alianza Editorial.
Rodrigo, M.J., Máiquez, M.L., Martín, J.C. y Byrne, S. (2008). Preservación Familiar: un enfoque positivo para la intervención con familias. Madrid: Pirámide.

Rodrigo, M.J., Máiquez, M.L., Martín, J.C y Rodríguez, G. (2006) El asesoramiento a familias con riesgo psicosocial. En C. Monereo y J.I. Pozo (Coords.) La práctica del asesoramiento educativo a examen (139-152). Barcelona: Graó.

Rodrigo, M.J., Martín, J.C., Cabrera, E. y Máiquez, M.L (2009). Competencias parentales y familias en situación de riesgo Psicosocial (en prensa en la revista de Intervención Psicosocial).

Rodrigo, M. J., Correa, A. D., Máiquez, M. L., Martín, J. C. y Rodríguez, G. (2006). Family preservation services in Canary Islands: Predictors of the efficacy of a Parenting program for families at-risk of social exclusión. European Psychologist, 11 (1), 57- 70.

Rodrigo, M.J., Máiquez, M.L., Correa, A.D., Martín, J.C. y Rodríguez, G. (2006). Outcome evaluation of a community centre-based program for mothers at high psychosocial risk. Child Abuse and Neglect, 39 (9), 1049-1064.

Rodrigo, M.J., Máiquez, M.L., Byrne, S., Rodríguez, B., Martín, J.C., Rodríguez, G. y Pérez, L. (2008). Programa Crecer Felices en Familia: programa de apoyo psicoeducativo para promover el desarrollo infantil. Valladolid. Junta de Castilla y León.

Vila, I. (1998). Intervención psicopedagógica en el contexto familiar. En M.J. Rodrigo y J. Palacios (Comps.). Familia y desarrollo humano. Madrid: Alianza.

Manuscrito recibido: 22/10/2009

Revisión recibida: 11/11/2009

Manuscrito aceptado: 13/11/2009 